\title{
Stowing not recommended when taking a capillary blood sample
}

Bo Fransen, Veerle Wille, Jolanda Maaskant

Emma Children's Hospital, Amsterdam UMC- Location AMC, Amsterdam, The Netherlands b.fransen@amc.uva.nl

\section{Introduction}

Capillary blood sampling is a procedure occurring in pediatric care frequently.

We noticed variation in care in the execution of this procedure: some nurses stow or massage the body part when taking a capillary blood sample, while others believe this may result in deviant laboratory results. Besides safety issues, this variation also results in ambiguous information for children and parents. Therefore, we systematically searched the literature that investigated different techniques of taking capillary blood samples in pediatric patients.

\section{Methods}

We researched PubMed and TRIP database for studies published between 2007 and 2017. No further limits were applied. The articles were selected on title, abstract and full-text according predefined inclusion and exclusion criteria. Tools of the Cochrane Collaboration were used for the quality assessment and data extraction.

\section{Results}

The search resulted in 4 low quality studies: 1 survey, 1 recommendation rapport and 2 guidelines. All studies report best practices.

The recommendations are congruent:

- It is believed that stowing or massaging the body part when taking a capillary blood sample may lead to deviant laboratory results. Besides that, stowing may lead to hemolysis, contamination of the blood flow with interstitial and intercellular fluid and impede blood flow.

- To get a capillary blood sample correctly and easy, it is recommended to warm the body part in order to stimulate the blood flow.

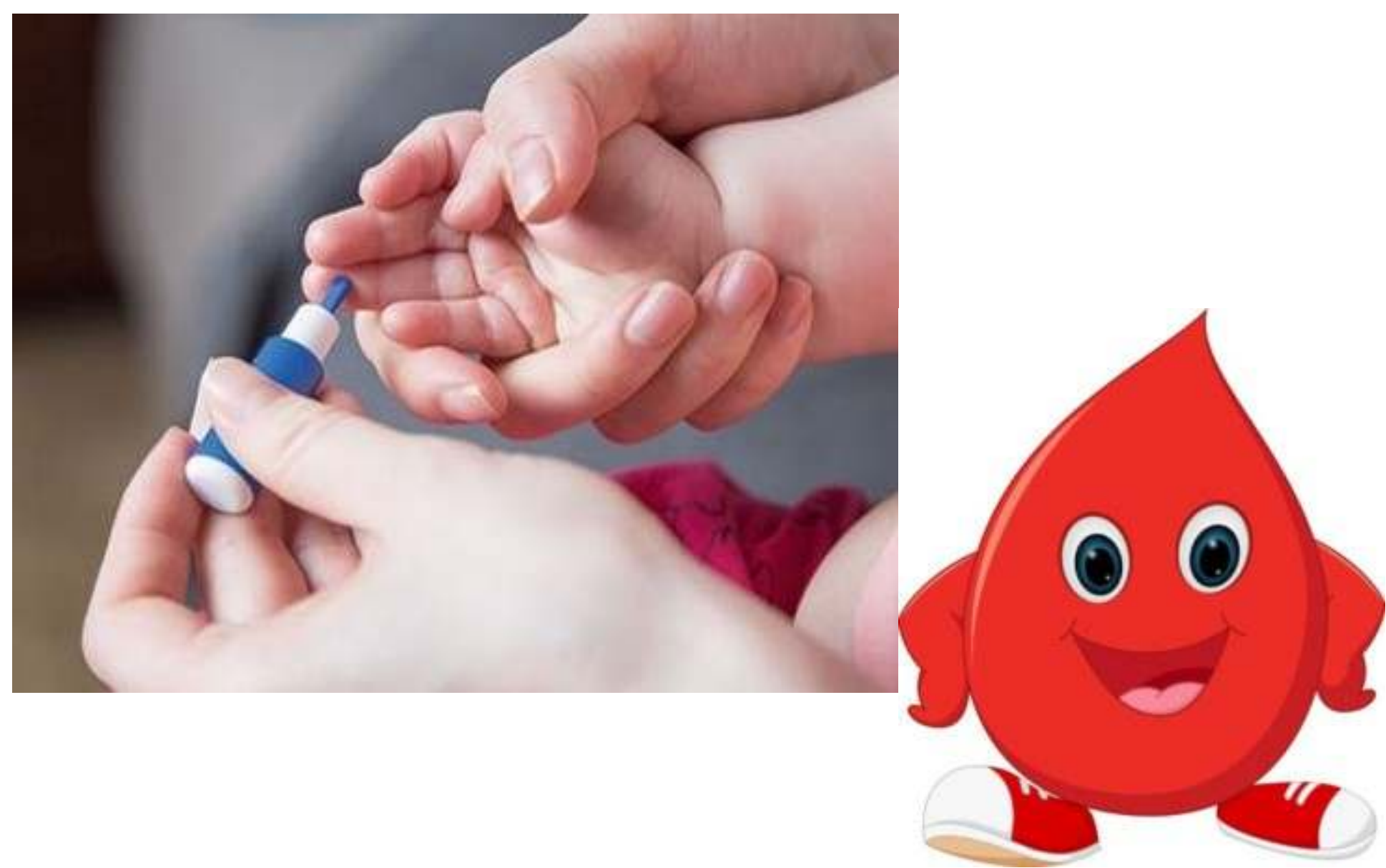

\section{Conclusion}

Strong evidence is lacking, but the recommendation from best practices is to avoid stowing or massaging the body part when taking a capillary blood sample in pediatric patients. 